\title{
Giant nonreciprocal magnetotransport in bulk trigonal superconductor $\mathrm{PbTaSe}_{2}$
}

\author{
T. Ideue $\odot,,^{1, *}, \dagger$ S. Koshikawa,,$^{1, \dagger}$ H. Namiki $\odot,{ }^{2}$ T. Sasagawa, ${ }^{2}$ and Y. Iwasa ${ }^{1,3}$ \\ ${ }^{1}$ Quantum-Phase Electronics Center (QPEC) and Department of Applied Physics, The University of Tokyo, Tokyo 113-8656, Japan \\ ${ }^{2}$ Laboratory for Materials and Structures, Tokyo Institute of Technology, Yokohama, Kanagawa 226-8503, Japan \\ ${ }^{3}$ RIKEN Center for Emergent Matter Science (CEMS), Wako, Saitama 351-0198, Japan
}

(Received 17 August 2020; revised 22 November 2020; accepted 8 December 2020; published 24 December 2020)

\begin{abstract}
We report giant bulk rectification in the bulk trigonal superconductor $\mathrm{PbTaSe}_{2}$. Nonreciprocal magnetotransport originating from a vortex ratchet motion was successfully observed, showing a significant enhancement during the superconducting transition, which is considered a model behavior for three-dimensional noncentrosymmetric superconductors with strong vortex pinning. Moreover, an unprecedented sign change of nonreciprocal transport was observed with increasing current, which indicates that the rectification effect can be tuned by the current value as well as the magnetic field. The present results represent the rich vortex dynamics and offer a principle for current control of rectification in noncentrosymmetric bulk superconductors.
\end{abstract}

DOI: 10.1103/PhysRevResearch.2.042046

\section{INTRODUCTION}

The noncentrosymmetric superconductor [1,2] is an attractive material platform owing to a variety of exotic quantum phenomena originating from the lattice symmetry breaking, such as the parity mixing of Cooper pairs [3], helical states [4], and Ising superconductivity [5,6]. Among them, nonreciprocal magnetotransport, which indicates the directional dichroism of the magnetoresistance or rectification effect under a magnetic field originating from broken inversion symmetry, has become recognized as an important probe for charge dynamics and also an emergent functionality in noncentrosymmetric conductors [7]. In noncentrosymmetric systems, the electrical resistance generally depends on the current direction under a magnetic field [8]. Such nonreciprocal magnetotransport has been studied in artificial helical structures or interfaces [9-13] and has recently been observed in bulk crystals [14-16]. In a polar semiconductor [14,15] and a chiral molecular conductor [16], a nonreciprocal signal originating from their crystal structures has been confirmed. It has been further clarified that intrinsic rectification effects can also be realized in noncentrosymmetric superconductors [17-24]. Thus far, only a limited number of examples including chiral $\mathrm{WS}_{2}$ nanotubes [17], two-dimensional superconductors such as $\mathrm{MoS}_{2}$ [18,22], MoGe/yttrium iron garnet (YIG) [19], $\mathrm{FeTe} / \mathrm{Bi}_{2} \mathrm{Te}_{3}$ [20], $\mathrm{SrTiO}_{3}$ [21], and $\mathrm{NbSe}_{2}$ [23] are known to show a rectification effect. In all cases, nonreciprocal magnetotransport is enhanced within the

\footnotetext{
*Corresponding author: ideue@ap.t.u-tokyo.ac.jp

${ }^{\dagger}$ These authors contributed equally to this work.
}

Published by the American Physical Society under the terms of the Creative Commons Attribution 4.0 International license. Further distribution of this work must maintain attribution to the author(s) and the published article's title, journal citation, and DOI. superconducting region, and possible origins such as the paraconductivity effect or vortex ratchet effect have been proposed. In addition, their relations with the pairing symmetry or quantum vortex dynamics are further discussed. However, the identification and classification of the detailed mechanism are still ongoing [24,25]. Moreover, because nonreciprocal superconducting transport has been discovered only in lowdimensional nanosystems with large fluctuations and weak vortex pinning and three-dimensional bulk crystals showing the nonreciprocal transport is missing, the observation of a superconducting rectification effect in noncentrosymmetric crystals in bulk forms is a promising way to further understand this phenomenon and electronic states/vortex dynamics in three-dimensional (3D) noncentrosymmetric superconductors.

In this Rapid Communication, we considered nonreciprocal magnetotransport in a noncentrosymmetric layerstructured superconductor $\mathrm{PbTaSe}_{2}$, which has trigonal symmetry similar to that of two-dimensional (2D) superconducting transition-metal dichalcogenides. The crystal structure, band structure, and electronic properties of $\mathrm{PbTaSe}_{2}$ have been well studied previously [26-33]. Owing to its unique noncentrosymmetric crystal symmetry [34] and large spin-orbit interaction, $\mathrm{PbTaSe}_{2}$ has attracted broad interest as a candidate of topological materials [26-33]. $\mathrm{PbTaSe}_{2}$ is also known to be a noncentrosymmetric superconductor with $T_{\mathrm{c}}=$ $3.7 \mathrm{~K}$. Anisotropic bulk superconducting behaviors with the anisotropy parameter of $H_{c 2}^{a b}(0) / H_{c 2}^{c}(0)=5$ were observed [33], where $H_{c 2}^{a b}(0)$ and $H_{c 2}^{c}(0)$ are the critical magnetic fields along the in-plane direction and out-of-plane direction, respectively. Figures 1(a) and 1(b) show the top [Fig. 1(a)] and side views [Fig. 1(b)] of the crystal structure of $\mathrm{PbTaSe}_{2}$. It can be seen as an alternating stacking of $\mathrm{Pb}$ and $\mathrm{TaSe}_{2}$ layers along the $c$ axis. One $\mathrm{TaSe}_{2}$ layer has trigonal symmetry similar to that of $2 \mathrm{D} \mathrm{MoS}_{2}$ or $\mathrm{NbSe}_{2}$, thus breaking the inversion symmetry along the in-plane direction [Fig. 1(a)]. Because the neighboring $\mathrm{TaSe}_{2}$ layers stack in the same 
(a)

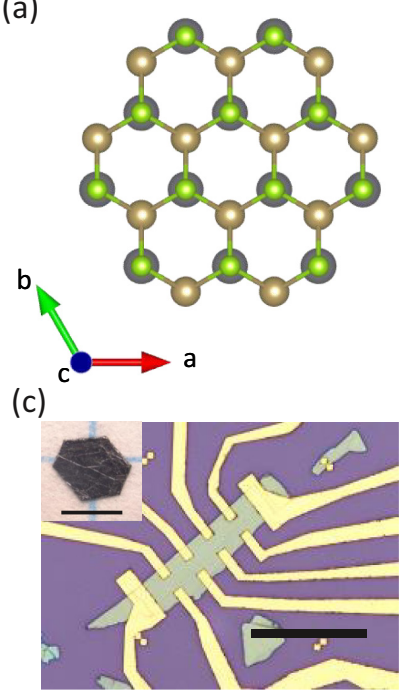

(b)

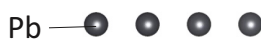

(d)
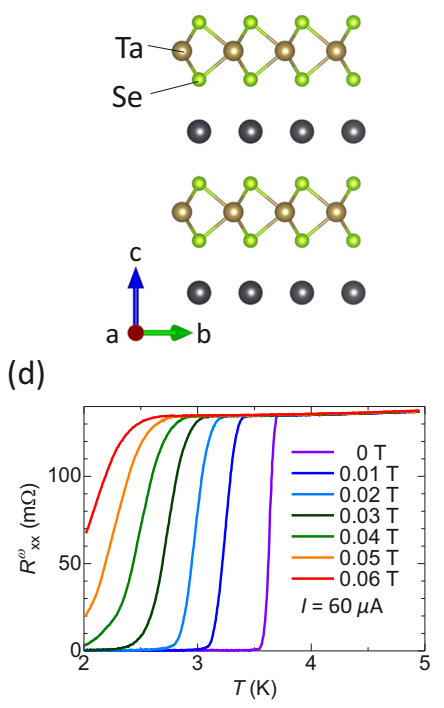

FIG. 1. Crystal structure and superconductivity of $\mathrm{PbTaSe}_{2}$. (a), (b) Crystal structure of $\mathrm{PbTaSe}_{2}$. (a) Top and (b) side views of $\mathrm{PbTaSe}_{2}$ crystal. (c) Image of $\mathrm{PbTaSe}_{2}$ device (device 1). Current flows along the longest axis of the exfoliated flake. Scale bar: $20 \mu \mathrm{m}$. The inset shows the bulk crystal of $\mathrm{PbTaSe}_{2}$. Scale bar: $1 \mathrm{~mm}$. (d) Temperature dependence of the first-harmonic resistance. Under a zero magnetic field, $R^{\omega}$ becomes zero at approximately $3.7 \mathrm{~K}$.

direction [Fig. 1(b)], the inversion symmetry is maintained to be broken even in multilayer bulk $\mathrm{PbTaSe}_{2}$, which is in significant contrast to $2 \mathrm{H}-\mathrm{MoS}_{2}$ with a centrosymmetric structure in multiple layers. A comparison of the electronic states of $\mathrm{PbTaSe}_{2}$ and metallic transition-metal dichalcogenides is also discussed. Transition-metal dichalcogenides such as $2 \mathrm{H}$ $\mathrm{NbSe}_{2}, \mathrm{TaS}_{2}$, and $\mathrm{TaSe}_{2}$ are known to reveal charge density wave (CDW) transitions, which can be tuned by various parameters [35-37]. In $\mathrm{PbTaSe}_{2}$, however, CDW instability is eliminated by the intercalation of the $\mathrm{Pb}$ layer [32].

Owing to its layered crystal structure, we can obtain monodomain $\mathrm{PbTaSe}_{2}$ flakes using an exfoliation method, and a large current density is realized in them. This is highly advantageous for the observation of nonreciprocal transport. In addition, the crystal structure of three-dimensional $\mathrm{PbTaSe}_{2}$, which is similar to that of two-dimensional $\mathrm{MoS}_{2}$ or $\mathrm{NbSe}_{2}$ $[18,22,23]$, offers an opportunity to compare the nonreciprocal magnetotransport of these trigonal crystals and study the effect of the dimensionality on the superconducting rectification effect. Thus, $\mathrm{PbTaSe}_{2}$ is considered to be an ideal candidate for studying bulk nonreciprocal magnetoresistance.

\section{RESULTS}

\section{A. Transport properties of $\mathrm{PbTaSe}_{2}$ devices}

We fabricated devices of exfoliated $\mathrm{PbTaSe}_{2}$ flakes with two different geometries (devices 1 and 2). The thickness of device 1 (device 2) is $80 \mathrm{~nm}(50 \mathrm{~nm})$, which is still regarded as bulk. The crystal orientations of the $\mathrm{PbTaSe}_{2}$ flakes were determined from the shape of the sample edge. According to a previous study [38], the straight line of exfoliated flakes of transition-metal dichalcogenides such as $\mathrm{MoS}_{2}$ corresponds to a zigzag edge. We expect that this conclusion can also be applicable to similar layered trigonal crystals of $\mathrm{PbTaSe}_{2}$. This claim for the crystal orientation was later confirmed in another experiment by scanning transmission electron microscope measurements [34]. Thus, the current is parallel to the zigzag direction in device 1 [Fig. 1(c)], whereas the current flows along the armchair direction in device 2 .

We first checked the basic electric properties of these devices through the first-harmonic components of the lock-in signals. Figure 1(d) shows the temperature dependence of the first-harmonic resistance $\left(R^{\omega} \equiv \frac{V^{\omega}}{I_{0}}\right.$, where $V^{\omega}$ and $I_{0}$ are the first-harmonic components of voltage in the lock-in measurement and current magnitude, respectively) of device 1 . Under a zero magnetic field, it shows a superconducting transition at approximately $T_{\mathrm{c}}=3.7 \mathrm{~K}$, which is consistent with previous reports for large bulk samples [33]. The sharp transition at approximately $T_{\mathrm{c}}$ under a zero magnetic field represents a typical superconducting transition in bulk crystals, indicating that the exfoliated flakes with several tens of nanometers can still be regarded as bulk crystals. By applying a magnetic field, the zero resistance is suppressed and the critical magnetic field at $T=2 \mathrm{~K}$ is approximately $B=0.06 \mathrm{~T}$ as obtained from the half value of the resistance in the normal state. It should be noted that the superconducting transition is broadened as the magnetic field increases. A similar broadening is frequently seen in layered superconductors, most dramatically in high $T_{\mathrm{c}}$ cuprates. This broadening is attributed to the vortex effect due to its anisotropic layered structure. We believe that this vortex effect is responsible for the nonreciprocal charge transport observed in this study.

\section{B. Nonreciprocal magnetotransport in $\mathrm{PbTaSe}_{2}$}

To study the nonreciprocal magnetotransport in this compound, we focused on the second-harmonic resistance $\left(R^{2 \omega} \equiv\right.$ $\frac{V^{2 \omega}}{I_{0}}$, where $V^{2 \omega}$ is the second-harmonic component of the voltage in the lock-in measurement). The second-harmonic ac measurement is generally used to probe the difference in the resistance depending on the current direction, thus representing nonreciprocal electric transport [10-23]. In Figs. 2(a)-2(c), we show schematic illustrations of the current direction [Fig. 2(a)], and the magnetic field dependence of $R^{\omega}$ [Fig. 2(b)] and $R^{2 \omega}$ [Fig. 2(c)], of device 1 at $T=2 \mathrm{~K}$. The current is set to $60 \mu \mathrm{A}$. With an increasing external magnetic field, the first-harmonic resistance changes from zero to a finite value of approximately $B=0.06 \mathrm{~T}$, which is consistent with the temperature dependence of the resistance under a magnetic field [Fig. 1(d)]. Accordingly, the longitudinal second-harmonic resistance $R_{\mathrm{xx}}^{2 \omega}$, which is first negligibly small in a zero-resistance state, shows a steep peak structure during this transition, and is suppressed again in a normal state in the high magnetic field region. This indicates that nonreciprocal transport is largely enhanced during the superconducting transition, similar to the previous cases of low-dimensional noncentrosymmetric superconductors [17-23]. However, the zero-resistance state under a magnetic field is extremely fragile in previous cases owing to the weak pinning of the vortices or quantum fluctuations, which are characteristics in low-dimensional systems. Thus, the peak structure of $R^{2 \omega}$ is 
(a)

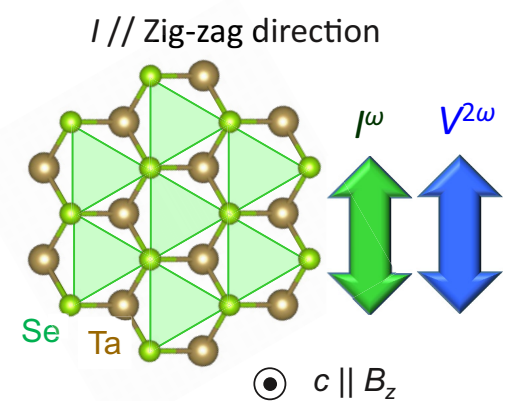

(d)

I // Armchair direction

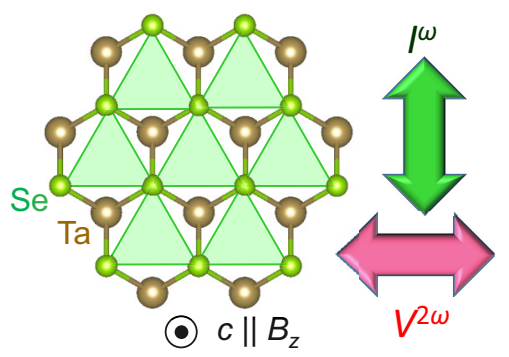

(b)

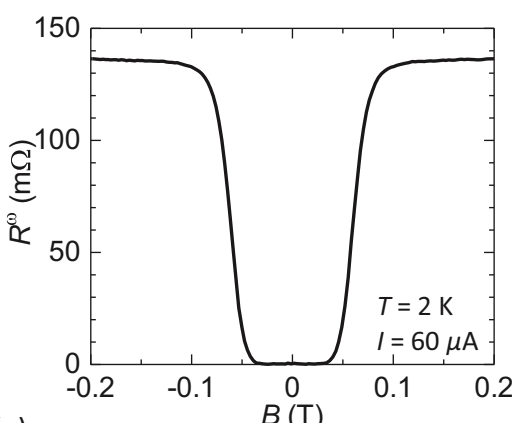

(e)

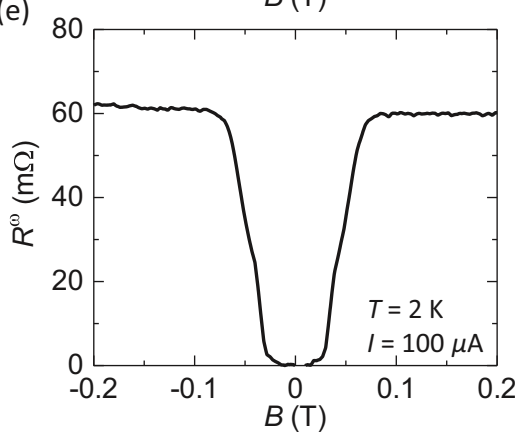

(c)

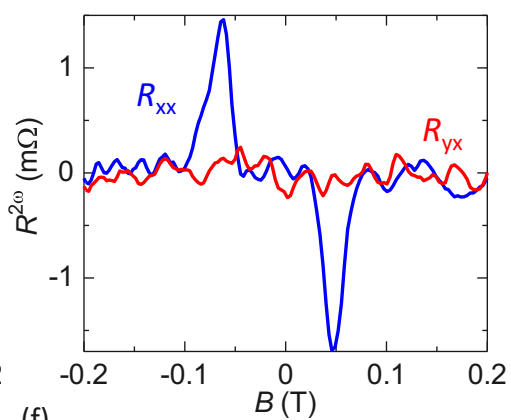

(17)

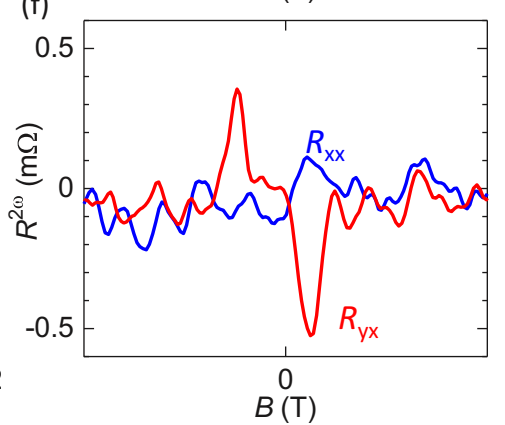

FIG. 2. Directional dependence of the second-harmonic magnetoresistance under two distinct device configurations. (a) Schematic illustration of device 1. Current flows along the zigzag direction. (b) First-harmonic resistance $R^{\omega}$ of device 1. (c) Second-harmonic resistance $R^{2 \omega}$ of device 1. $R_{\mathrm{xx}}^{2 \omega}$ and $R_{\mathrm{yx}}^{2 \omega}$ represent longitudinal and transverse signals, respectively. Peak structures around the superconducting transition appear in $R_{\mathrm{xx}}^{2 \omega}$. (d) Schematic illustration of device 2. Current flows along the armchair direction. (e) $R^{\omega}$ of device 2 . (f) $R_{\mathrm{xx}}^{2 \omega}$ and $R_{\mathrm{yx}}^{2 \omega}$ signals of device 2. Peak structures can be observed in $R_{\mathrm{yx}}^{2 \omega}$.

broad, reflecting the gradual transition from a state of zero resistance to a normal state in previous reports [17-23]. By contrast, in the present case of $\mathrm{PbTaSe}_{2}$, the superconducting transition is sharp and a steep peak structure appears near the critical magnetic field, manifesting the model behavior of the nonreciprocal magnetotransport in noncentrosymmetric bulk crystals. The transverse second-harmonic signal $R_{\mathrm{yx}}^{2 \omega}$ is close to zero in this configuration [Fig. 2(c)], which agrees with the trigonal symmetry and expected current direction.

Because a vortex flow is the main origin of the emergence of finite resistance under the present condition, we can attribute the observed nonreciprocal electric transport to the vortex ratchet motion by the asymmetric pinning potential $[22,25]$. Note that the magnitude of the rectification effect is significant in this compound. The second-harmonic resistance normalized by the first-harmonic resistance and the current density $i$, that is, the value of $\frac{R^{2 \omega}}{R^{\omega} i}$, is approximately $5.0 \times 10^{-10} \mathrm{~m}^{2} \mathrm{~A}^{-1}$, which is far larger than that of $2 \mathrm{D}$ trigonal superconductor $\operatorname{MoS}_{2}\left(\frac{R^{2 \omega}}{R^{\omega} i} \sim 6.0 \times 10^{-12} \mathrm{~m}^{2} \mathrm{~A}^{-1}\right)$ with a similar crystal symmetry [22].

Nonreciprocal superconducting transport was first discovered in gate-induced 2D superconductivity in $\mathrm{MoS}_{2}$ [18], which has a trigonal structure, the same as $\mathrm{PbTaSe}_{2}$. It is well known that, in such highly crystalline 2D superconductors, the superconductivity occurs in a monolayer limit, and the vortex pinning effect is rather weak. Therefore, in conjunction with the large 2D fluctuation effect, the vortex liquid state can be a ground state $[39,40]$, where the vortex motion is attributed to quantum mechanical tunneling creep motion. Thus this state is called a quantum metal or failed superconductor [41]. Recently, it was found that the nonreciprocal response in this quantum metallic state, where vortex flow occurs via a quantum mechanical tunneling, is diminishingly small, while it is enhanced in the classical vortex flow region [22]. Since the vortex motion in 3D $\mathrm{PbTaSe}_{2}$ is also in the classical regime, the present result indicates that an enhanced nonreciprocal response is highly likely attributed to the larger pinning effect of the 3D superconductor $\mathrm{PbTaSe}_{2}$ than that in the 2D superconductor, gated $\mathrm{MoS}_{2}$. It should also be noted that noncentrosymmetric superconductors can have complex pinning states owing to the formation of a fractional vortex or time-reversal symmetry breaking, which might affect the above ratchet motion of vortices [42-44].

To further confirm the intrinsic nature of the observed nonreciprocal magnetotransport, we also investigated the nonreciprocal transport of device 2 [Figs. 2(d)-2(f)] at $T=2 \mathrm{~K}$, in which the current flows along the armchair direction. The current is set to $100 \mu \mathrm{A}$. Although $R^{\omega}$ shows a similar magnetic field dependence as device 1 [Fig. 2(e)], the behaviors of $R_{\mathrm{xx}}^{2 \omega}$ and $R_{\mathrm{yx}}^{2 \omega}$ are opposite between devices 1 and 2; a large enhancement of the signal around the transition from the superconducting phase to the normal phase was observed as $R_{\mathrm{yx}}^{2 \omega}$ instead of $R_{\mathrm{xx}}^{2 \omega}$, and the signals in $R_{\mathrm{xx}}^{2 \omega}$ were absent. This characteristic presence or absence of $R_{\mathrm{xx}}^{2 \omega}$ and $R_{\mathrm{yx}}^{2 \omega}$ signals has also been observed in a $2 \mathrm{D}$ trigonal superconductor $\mathrm{MoS}_{2}$ [22], indicating that signals unambiguously originate from a trigonal lattice symmetry. 
(a)

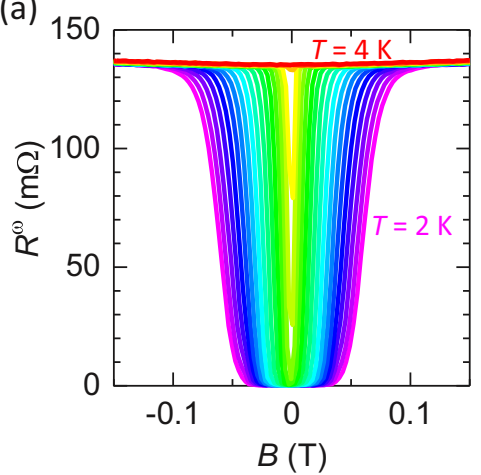

(b)

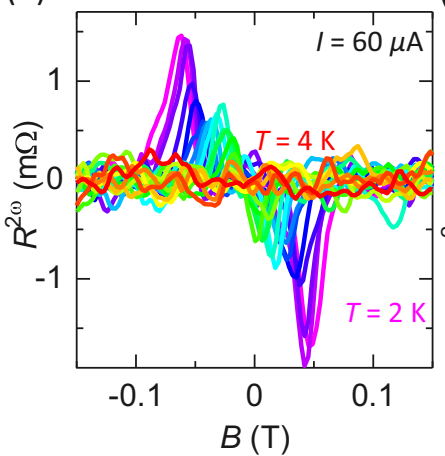

(c)

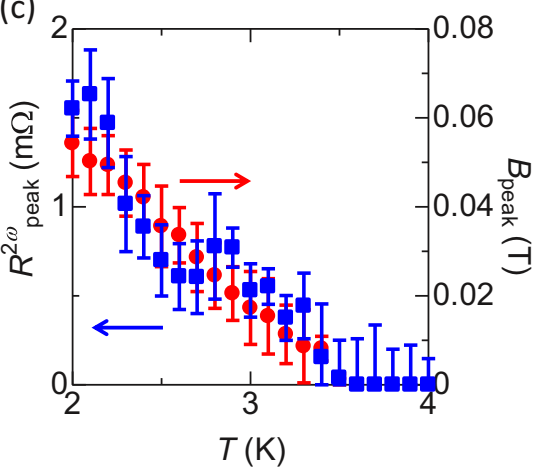

FIG. 3. Temperature dependence of the nonreciprocal magnetoresistance. (a) $R_{\mathrm{xx}}^{\omega}$ and (b) $R_{\mathrm{xx}}^{2 \omega}$ signals of device 1 at various temperatures under a current of $60 \mu \mathrm{A}$. The temperature was varied in 0.1-K steps from 2 to $4 \mathrm{~K}$. By increasing the temperature, the critical magnetic field in $R_{\mathrm{xx}}^{\omega}$ and the corresponding peak of $R_{\mathrm{xx}}^{2 \omega}$ shift to the lower magnetic field. (c) Temperature variations of peak positions (red circles) and peak values (blue) of $R_{\mathrm{xx}}^{2 \omega}$. Error bars are estimated by the fluctuation values of peaks and background signals. Peak structures of $R_{\mathrm{xx}}^{2 \omega}$ developed in the superconducting transition region. There is a good correspondence between the peak positions and peak values.

Next, we focus on the temperature dependence of the rectification effect. The magnetic field dependences of $R_{\mathrm{xx}}^{\omega}$ and $R_{\mathrm{xx}}^{2 \omega}$ of device 1 at various temperatures are displayed in Figs. 3(a) and 3(b), respectively. As the temperature increases, the zero-resistance state shrinks, which causes a decrease in the critical magnetic field. Correspondingly, the peak position in $R_{\mathrm{xx}}^{2 \omega}$, which mostly coincides with the critical magnetic field in $R_{\mathrm{xx}}^{\omega}$, shifts to a lower magnetic field. In Fig. 3(c), we plot the peak positions and peak values of $R_{\mathrm{xx}}^{2 \omega}$ as a function of temperature. Here, $R_{\mathrm{xx}}^{2 \omega}$ is developed at below $T_{\mathrm{c}}$, clearly demonstrating the enhancement of nonreciprocal magnetotransport in the superconducting state. In addition, we can recognize the close correspondence between the peak value of $R_{\mathrm{xx}}^{2 \omega}$ and the peak magnetic field. This indicates that the (a)

(d)
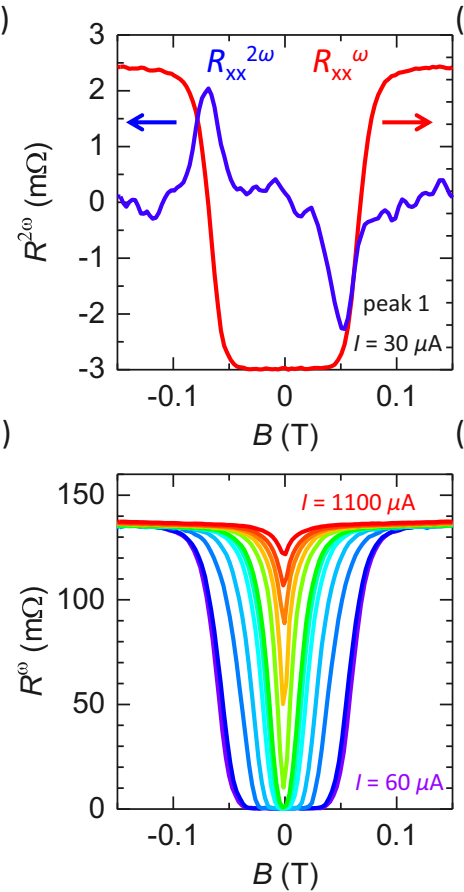

(b)
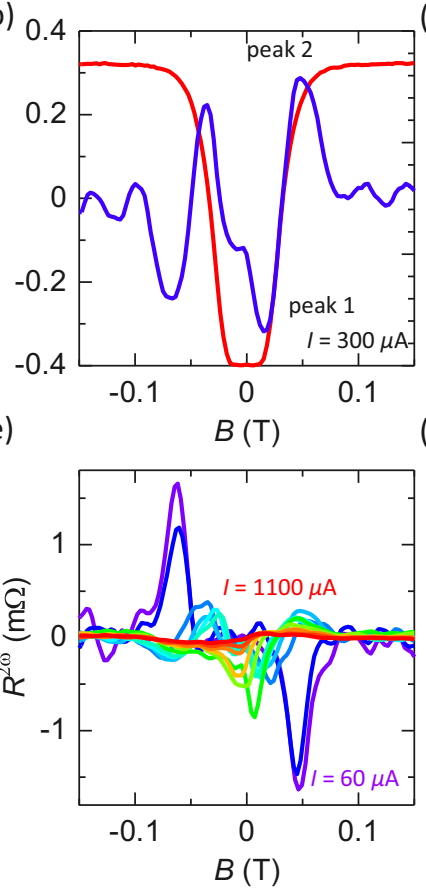

(c)
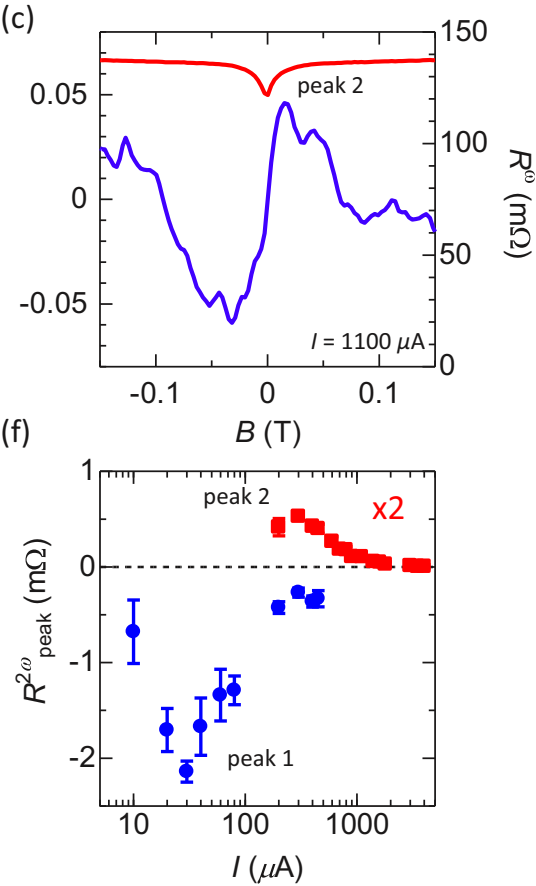

FIG. 4. Current dependence and sign reversal of nonreciprocal magnetoresistance. (a)-(c) Magnetic field dependence of $R_{\mathrm{xx}}^{\omega}$ (red) and $R_{\mathrm{xx}}^{2 \omega}$ (blue) of device 1 at $2 \mathrm{~K}$ under a current of (a) $30 \mu \mathrm{A}$, (b) $300 \mu \mathrm{A}$, and (c) $1100 \mu \mathrm{A}$, respectively. Only a negative peak (peak 1) can be observed in a low-current region such as $I=30 \mu \mathrm{A}$. As we increase the current (for example, when $I=300 \mu \mathrm{A}$ ), two peaks with opposite signs (peaks 1 and 2) become comparable. When the current is increased up to $I=1100 \mu \mathrm{A}$, a positive signal (peak 2) becomes dominant. (d), (e) Magnetic field dependence of (d) $R_{\mathrm{xx}}^{\omega}$ and (e) $R_{\mathrm{xx}}^{2 \omega}$ of device 1 at $2 \mathrm{~K}$ under various current values. Data of $I=60,80,200,300,400$, 450, 500, 600, 700, 800, 900, and $1100 \mu \mathrm{A}$ are plotted. (f) Current dependence of the negative (blue) and positive (red) peak values. Positive signals are multiplied by 2 to make them clearly distinguishable. Error bars are estimated by the fluctuation values of the peaks and background signals. In the low-current region, negative signals are dominant, whereas positive nonreciprocal signals develop by increasing the current. 
magnitude of nonreciprocal transport at each peak position is proportional to the magnetic field, that is, the number of vortices, which is consistent with the above scenario of the vortex ratchet effect.

Finally, we investigated the detailed current dependence of $R_{\mathrm{xx}}^{2 \omega}$ and found an unprecedented sign change of nonreciprocal transport with increasing current. In Figs. 4(a)-4(c), we show the magnetic field dependences of $R_{\mathrm{xx}}^{\omega}$ (red) and $R_{\mathrm{xx}}^{2 \omega}$ (blue) under a current of 30, 300, and $1100 \mu \mathrm{A}$, respectively, as representative examples. In the low-current region [ $I=30 \mu \mathrm{A}$; Fig. 4(a)], both $R_{\mathrm{xx}}^{\omega}$ and $R_{\mathrm{xx}}^{2 \omega}$ show similar behavior to that in Fig. 2(c). If we increase the current, the zero-resistance state in $R_{\mathrm{xx}}^{\omega}$ becomes narrower and the peak of $R_{\mathrm{xx}}^{2 \omega}$ (peak 1) shifts to a lower magnetic field with its magnitude being suppressed similarly to the temperature dependence of Figs. 3(a) and 3(b). At the same time, we observed another peak structure of $R_{\mathrm{xx}}^{2 \omega}$ (peak 2) with the opposite sign in a higher magnetic field region under a large current [Figs. 4(b) and 4(c)]. The magnitude of the newly emerged positive signals becomes comparable to that of the original negative signals under $I=300 \mu \mathrm{A}$ [Fig. 4(b)], and finally dominates $R_{\mathrm{xx}}^{2 \omega}$ under $I=1100 \mu \mathrm{A}$ [Fig. 4(c)]. In Figs. 4(d) and 4(e), we summarize $R_{\mathrm{xx}}^{\omega}$ and $R_{\mathrm{xx}}^{2 \omega}$ of device 1 at $T=2 \mathrm{~K}$ under various current densities. Figure 4(f) shows the peak values of each signal as a function of the current. This clearly indicates that there are two distinct contributions to nonreciprocal magnetotransport, which become dominant in different current or magnetic field regions.

\section{DISCUSSION}

In previous studies on superconducting films with artificially made asymmetric potentials, the sign-reversible superconducting rectification effect has been observed [45-48]. The observed change in sign of the rectification effect was attributed to the many-body effect of the vortices [45-48]. In the present study, a similar effect of interacting vortices might contribute to the current-induced change in sign of $R_{\mathrm{xx}}^{2 \omega}$. In the low-current region, only weakly pinned vortices flow to avoid strongly pinned vortices, causing large nonreciprocal magnetotransport. However, under a large current, all vortices, including those originally pinned in the low-current region, are driven by the Lorentz force, where the vortex motion is affected by the vortex-vortex interaction and possibly leads to a sign reversal of the rectification effect. By contrast, we note that the value of $R_{\mathrm{xx}}^{\omega}$, in the region where a positive nonreciprocal magnetotransport signal appears, is larger than half the values of the normal state. The effect of the amplitude fluctuation of the superconducting order parameter, which is discussed in the paraconductivity region [18], may not be neglected in such a high resistance region under a normal state.

\section{CONCLUSION}

In summary, we observed nonreciprocal magnetotransport in the noncentrosymmetric bulk superconductor $\mathrm{PbTaSe}_{2}$. The second-harmonic magnetoresistance corresponding to the intrinsic rectification effect is largely enhanced during the superconducting transition and suppressed under zeroresistance and normal states, which can be regarded as textbook behavior in noncentrosymmetric bulk superconductors. A vortex ratchet motion with a large pinning potential is a plausible origin. By increasing the current, a change in sign of the rectification effect was observed, potentially reflecting the crossover of the nonreciprocal magnetotransport of different origins. The present results offer an effective method of probing the vortex dynamics and current control in noncentrosymmetric bulk superconductors. The study of nonreciprocal magnetotransport in other noncentrosymmetric 3D superconductors and the search for different mechanisms of nonreciprocal magnetotransport are important future topics.

\section{ACKNOWLEDGMENTS}

The authors thank N. Nagaosa, K. Hamamoto, R. Wakatsuki, Y. Saito, S. Hoshino, Y. M. Itahashi, and T. Nojima for the fruitful discussions. This work was supported by JSPS KAKENHI Grants No. JP19H05602, No. JP19K21843, No. JP19H01819, and No. JP20H05264, A3 Foresight Program, Yazaki Memorial Foundation for Science and Technology, and the JST PRESTO Grant No. JPMJPR19L1, and JST CREST Grant No. JPMJCR16F2.
[1] E. Bauer and M. Sigrist, Non-Centrosymmetric Superconductors: Introduction and Overview (Springer, Berlin, 2012).

[2] M. Smidman, M. B. Salamon, H. Q. Yuan, and D. F. Agterberg, Superconductivity and spin-orbit coupling in noncentrosymmetric materials: A review, Rep. Prog. Phys. 80, 036501 (2017).

[3] L. P. Gor'kov and E. I. Rashba, Superconducting 2D System with Lifted Spin Degeneracy: Mixed Singlet-Triplet State, Phys. Rev. Lett. 87, 037004 (2001).

[4] V. P. Mineev and K. V. Samokhin, Helical phases in superconductors, J. Exp. Theor. Phys. 78, 401 (1994).

[5] J. M. Lu, O. Zheliuk, I. Leermakers, N. F. Q. Yuan, U. Zeitler, K. T. Law, and J. T. Ye, Evidence for two-dimensional Ising superconductivity in gated $\mathrm{MoS}_{2}$, Science 350, 1353 (2015).
[6] Y. Saito, Y. Nakamura, M. S. Baharamy, Y. Kohama, J. Ye, Y. Kasahara, Y. Nakagawa, M. Onga, M. Tokunaga, T. Nojima, Y. Yanase, and Y. Iwasa, Superconductivity protected by spinvalley locking in ion-gated $\mathrm{MoS}_{2}$, Nat. Phys. 12, 144 (2016).

[7] Y. Tokura and N. Nagaosa, Nonreciprocal responses from noncentrosymmetric quantum materials, Nat. Commun. 9, 3740 (2018).

[8] G. L. J. A. Rikken, J. Fölling, and P. Wyder, Electrical Magnetochiral Anisotropy, Phys. Rev. Lett. 87, 236602 (2001).

[9] G. L. J. A. Rikken and P. Wyder, Magnetoelectric Anisotropy in Diffusive Transport, Phys. Rev. Lett. 94, 016601 (2005).

[10] C. O. Avci, K. Garello, A. Ghosh, M. Gabureac, S. F. Alvarado, and P. Gambardella, Unidirectional spin Hall magnetoresistance in ferromagnet/normal metal bilayers, Nat. Phys. 11, 570 (2015). 
[11] P. He, S. S.-L. Zhang, D. Zhu, Y. Liu, Y. Wang, J. Yu, G. Vlgnale, and H. Yang, Bilinear magnetoelectric resistance as a probe of three-dimensional spin texture in topological surface states, Nat. Phys. 14, 495 (2018).

[12] P. He, S. M. Walker, S. S.-L. Zhang, F. Y. Bruno, M. S. Baharamy, J. M. Lee, R. Ramaswamy, K. Cai, O. Heinonen, G. Vignale, F. Baumberger, and H. Yang, Observation of Out-ofPlane Spin Texture in a $\mathrm{SrTiO}_{3}(111)$ Two-Dimensional Electron Gas, Phys. Rev. Lett. 120, 266802 (2018).

[13] D. Choe, M.-J. Jin, S.-1. Kim, H.-J. Choi, J. Jo, I. Oh, J. Park, H. Jin, H. C. Koo, B.-C. Min, S. Hong, H.-W. Lee, S.-H. Baek, and J.-W. Yoo, Gate-tunable giant nonreciprocal charge transport in noncentrosymmetric oxide interfaces, Nat. Commun. 10, 4510 (2019).

[14] T. Ideue, K. Hamamoto, S. Koshikawa, M. Ezawa, S. Shimizu, Y. Kaneko, Y. Tokura, N. Nagaosa, and Y. Iwasa, Bulk rectification effect in a polar semiconductor, Nat. Phys. 13, 578 (2017).

[15] P. He, C.-H. Hsu, S. Shi, K. Cai, J. Wang, Q. Wang, G. Eda, H. Lin, V. M. Pereira, and H. Yang, Nonlinear magnetotransport shaped by Fermi surface topology and convexity, Nat. Commun. 10, 1290 (2019).

[16] F. Pop, P. Auban-Senzier, E. Canadell, G. L. J. A. Rikken, and N. Avarvari, Electrical magnetochiral anisotropy in a bulk chiral molecular conductor, Nat. Commun. 5, 3757 (2014).

[17] F. Qin, W. Shi, T. Ideue, M. Yoshida, A. Zak, R. Tennne, T. Kikitsu, D. Inoue, D. Hashizume, and Y. Iwasa, Superconductivity in a chiral nanotube, Nat. Commun. 8, 14465 (2017).

[18] R. Wakatsuki, Y. Saito, S. Hoshino, Y. M. Itahashi, T. Ideue, M. Ezawa, Y. Iwasa, and N. Nagaosa, Nonreciprocal charge transport in noncentrosymmetric superconductors, Sci. Adv. 3, e1602390 (2017).

[19] J. Lustikova, Y. Shiomi, N. Yokoi, N. Kabeya, N. Kimura, K. Ienaga, S. Kaneko, S. Okuma, S. Takahashi, and E. Saitho, Vortex rectenna powered by environmental fluctuations, Nat. Commun. 9, 4922 (2018).

[20] K. Yasuda, H. Yasuda, T. Liang, R. Yoshimi, A. Tsukazaki, K. S. Takahashi, N. Nagaosa, M. Kawasaki, and Y. Tokura, Nonreciprocal charge transport at topological inuslator/superconductor interface, Nat. Commun. 10, 2734 (2019).

[21] Y. M. Itahashi, T. Ideue, Y. Saito, S. Shimizu, T. Ouchi, T. Nojima, and Y. Iwasa, Nonreciprocal transport in gate-induced polar superconductor $\mathrm{SrTiO}_{3}$, Sci. Adv. 6, eaay9120 (2020).

[22] Y. M. Itahashi, Y. Saito, T. Ideue, T. Nojima, and Y. Iwasa, Quantum and classical ratchet motions of vortices in a $2 \mathrm{D}$ trigonal superconductor, Phys. Rev. Research 2, 023127 (2020).

[23] E. Zhang, X. Xu, Y.-C. Zhou, L. Ai, X. Dong, C. Huang, P. Leng, S. Liu, Y. Zhang, Z. Jia, X. Peng, M. Zhao, Y. Yang, Z. Li, H. Guo, S. J. Haigh, N. Nagaosa, J. Shen, and F. Xiu, Nonreciprocal superconducting $\mathrm{NbSe}_{2}$ antenna, Nat. Commun. 11, 5634 (2020).

[24] R. Wakatsuki and N. Nagaosa, Nonreciprocal Current in Noncentrosymmetric Rashba Superconductors, Phys. Rev. Lett. 121, 026601 (2018).

[25] S. Hoshino, R. Wakatsuki, K. Hamamoto, and N. Nagaosa, Nonreciprocal charge transport in two-dimensional noncentrosymmetric superconductors, Phys. Rev. B 98, 054510 (2018).

[26] M. N. Ali, Q. D. Gibson, T. Klimczuk, and R. J. Cava, Noncentrosymmetric superconductor with a bulk three-dimensional
Dirac cone gapped by strong spin-orbit coupling, Phys. Rev. B 89, 020505(R) (2014).

[27] G. Bian, T.-R. Chang, R. Sankar, S.-Y. Xu, H. Zheng, T Neupert, C.-K. Chiu, S.-M. Huang, G. Chang, I. Belopolski, D. S. Sanchez, M. Neupane, N. Alidoust, C. Liu, B. Wang, C.-C. Lee, H.-T. Jeng, C. Zhang, Z. Yuan, S. Jia et al., Topological nodal-line fermions in spin-orbit metal $\mathrm{PbTaSe}_{2}$, Nat. Commun. 7, 10556 (2015).

[28] S.-Y. Guan, P.-J. Chen, M.-W. Chu, R. Sankar, F. Chou, H.-T. Jeng, C.-S. Chang, and T.-M. Chuang, Superconducting topological surface states in the noncentrosymmetric bulk superconductor $\mathrm{PbTaSe}_{2}$, Sci. Adv. 2, e1600894 (2016).

[29] M. X. Wang, Y. Xu, L. P. He, J. Zhang, X. C. Hong, P. L. Cai, Z. B. Wang, J. K. Dong, and S. Y. Li, Nodeless superconducting gaps in noncentrosymmetric superconductor $\mathrm{PbTaSe}_{2}$ with topological bulk nodal lines, Phys. Rev. B 93, 020503(R) (2016).

[30] C.-L. Zhang, Z. Yuan, G. Bian, S.-Y. Xu, X. Zhang, M. Z. Hasan, and S. Jia, Superconducting properties in single crystals of the topological nodal semimetal $\mathrm{PbTaSe}_{2}$, Phys. Rev. B 93, 054520 (2016).

[31] G. M. Pang, M. Smidman, L. X. Zhao, Y. F. Wang, Z. F. Weng, L. Q. Che, Y. Chen, X. Lu, G. F. Chen, and H. Q. Yuan, Nodeless superconductivity in noncentrosymmetric $\mathrm{PbTaSe}_{2}$ single crystals, Phys. Rev. B 93, 060506(R) (2016).

[32] T.-R. Chang, P.-J. Chen, G. Bian, S.-M. Huang, H. Zheng, T. Neupert, R. Sankar, S.-Y. Xu, I. Belopolski, G. Chang, B. Wang, F. Chou, A. Bansil, H.-T. Jeng, H. Lin, and M. Z. Hasan, Topological Dirac surface states and superconducting paring correlations in $\mathrm{PbTaSe}_{2}$, Phys. Rev. B 93, 245130 (2016).

[33] H. Namiki and T. Sasagawa, Anisotropic superconducting properties of noncentrosymmetric $\mathrm{PbTaSe}_{2}$ as a candidate exotic superconductor, Sci. Adv. Mater. 8, 2097 (2016).

[34] See Supplemental Material at http://link.aps.org/supplemental/ 10.1103/PhysRevResearch.2.042046 for sample characterization and experimental details.

[35] M. Abdel-Hafiez, X. M. Zhao, A. A. Kordyuk, Y.-W. Fang, B. Pan, Z. He, C.-G. Duan, J. Zhao, and X. J. Chen, Enhancement of superconductivity under pressure and the magnetic phase diagram of tantalum disulfide single crystals, Sci. Rep. 6, 31824 (2016).

[36] A. Majumdar, D. VanGennep, J. Brisbois, D. Chareev, A. V. Sadakov, A. S. Usoltsev, M. Mito, A. V. Silhanek, T. Sarkar, A. Hassan, O. Karis, R. Ahuja, and M. Abdel-Hafiez, Interplay of charge density wave and multiband superconductivity in layered quasi-two-dimensional materials: The case of $2 \mathrm{H}-\mathrm{NbS}_{2}$ and $2 \mathrm{H}-\mathrm{NbSe}_{2}$, Phys. Rev. Mater. 4, 084005 (2020).

[37] Y. Kvashnin, D. VanGennep, M. Mito, S. A. Medvedev, R. Thiyagarajan, O. Karis, A. N. Vasiliev, O. Eriksson, and M. Abdel-Hafiez, Coexistence of Superconductivity and Charge Density Waves in Tantalum Disulfide: Experiment and Theory, Phys. Rev. Lett. 125, 186401 (2020).

[38] Y. Guo, C. Liu, Q. Yin, C. Wei, S. Lin, T. B. Hoffman, Y. Zhao, J. H. Edgar, Q. Chen, S. P. Lau, J. Dai, H. Yao. H.-S. P. Wong, and Y. Chai, Distinctive in-plane cleavage behaviors of two-dimensional layered materials, ACS Nano 10, 8980 (2016).

[39] Y. Saito, T. Nojima, and Y. Iwasa, Highly crystalline 2D superconductors, Nat. Rev. Mater. 2, 16094 (2016). 
[40] Y. Saito, Y. Kasahara, J. Ye, Y. Iwasa, and T. Nojima, Metallic ground state in an ion-gated two-dimensional superconductor, Science 350, 409 (2015).

[41] A. Kapitulnik, S. A. Kivelson, and B. Spivak, Anomalous metals: Failed superconductors, Rev. Mod. Phys. 91, 011002 (2019).

[42] M. Sigrist and D. F. Agterberg, The role of domain walls on the vortex creep dynamics in unconventional superconductors, Prog. Theor. Phys. 102, 965 (1999).

[43] C. Iniotakis, S. Fujimoto, and M. Sigrist, Fractional flux quanta at intrinsic metallic interfaces of noncentrosymmetric superconductors, J. Phys. Soc. Jpn. 77, 083701 (2008).

[44] E. Dumont and A. C. Mota, Unconventional vortex dynamics in superconducting states with broken time-reversal symmetry, Phys. Rev. B 65, 144519 (2002).
[45] J. E. Villegas, S. Savel'ev, F. Nori, E. M. Gonzalez, J. V. Anguita, R. García, and J. L. Vicent, A superconducting reversible rectifier that controls the motion of magnetic flux quanta, Science 302, 1188 (2003).

[46] J. E. Villegas, E. M. Gonzalez, M. P. Gonzalez, J. V. Anguita, and J. L. Vicent, Experimental ratchet effect in superconducting films with periodic arrays of asymmetric potentials, Phys. Rev. B 71, 024519 (2005).

[47] C. C. de Souza Silva, J. Van de Vondel, M. Morelle, and V. V. Moshchalkov, Controlled multiple reversals of a ratchet effect, Nature (London) 440, 651 (2006).

[48] Q. Lu, C. J. O. Reichhardt, and C. Reichhardt, Reversible vortex ratchet effects and ordeing in superconductors with simple asymmetric potential arrays, Phys. Rev. B 75, 054502 (2007). 